\title{
The Role of Systems Biology in Predictive Health and Personalized Medi- cine
}

\author{
Eberhard O. Voit ${ }^{*}, 1$ and Kenneth L. Brigham ${ }^{2}$ \\ ${ }^{I}$ Integrative BioSystems Institute, Georgia Institute of Technology, Atlanta, GA, USA \\ ${ }^{2}$ McKelvey Lung Transplant Center, Emory University Medical School, Atlanta, GA, USA
}

\begin{abstract}
Increasing health care costs mandate a shift in our thinking about health and disease from treatment to prevention. Effective prevention requires that we improve our abilities to predict and personalize health and disease risks. The article proposes that systems biology will play an essential role in personalized health assessments.
\end{abstract}

\section{INTRODUCTION}

It is no secret that the cost of health care in the U.S is staggering and the rate of increase outpaces inflation, consuming an ever increasing percentage of the gross domestic product. Novel and refined tools focused on diagnosis and treatment of disease will continue to amplify the individual and social financial burden. It is timely to contemplate fundamentally new approaches. While improvements in the efficiency of health care delivery could reduce costs, such effects will be incremental at best. The key to containing cost while improving human health is keeping people healthy. This radical change in focus of health care can be stimulated by government agencies and the media, but will ultimately depend on realigned thinking of health providers and healthy people.

Humans often judge general and personal risks differently, and make decisions accordingly. We tend to ignore risks to the general population, while accepting a personal regimen when we are convinced of a specific personal health risk. Switching perception from elusive population risks to scientifically predicted, personal risk is one of the challenges to creating a health focused system. Recognizing the importance of these health risk perceptions, the Director of the National Institutes of Health has called for investigations of the four $P s$-predictive, personalized, preventive medicine, along with participation by the public.

Establishing "personalized health profiles" is a challenging task. In some cases of clearly inherited diseases, risk profiles have been used for genetic counseling, although with restricted scope. Also, in the face of metabolically welldefined diseases such as phenylketonuria, parents are advised successfully to adjust the diets of their affected children. However, most complex diseases are difficult to predict, even when risk factors are known. Not all heavy smokers get lung cancer and some people who eat primarily red meat live to be centenarians. One standard explanation for carcinogenesis is a two-hit model, where a genetically predisposed person must be exposed to a carcinogenic environ-

*Address correspondence to this author at the Integrative BioSystems Institute, Georgia Institute of Technology, Atlanta, GA, USA;

E-mail: eberhard.voit@bme.gatech.edu ment to develop the disease. But, the majority of diseases seem to be a consequence of the confluence of multiple factors. Largely undefined combinations of genetic predisposition, lifestyle, and a variety of exposures appear to determine an individual's health, even though each "risk factor" alone may have no effect.

\section{SYSTEMS BIOLOGY}

The characterization of combinations of subtle changes in genetic and metabolic make-up will eventually require systems biology. There is no need here to define systems biology precisely. Suffice it to say that systems biology contains three sub-domains: (1) high-throughput experimentation, combined with bioinformatic evaluation methods; (2) technology development enabling this type of experimentation, including in vivo sensing and imaging; and (3) mathematical and computational modeling. Much has been written about the experimental and technological domains (1) and (2), and it is quite evident that massive amounts of genomic, proteomic, and metabolomic data will be crucial for assessing the health status of an individual [1-4]. By contrast, the role of computational systems biology in the prediction and personalization of health risk is not as obvious.

Studying complex diseases involves two approachesepidemiology and molecular biology. The former quantifies the significance of associations between risk factors and disease, while the latter targets causation at the biochemical or physiological level (Fig. 1). Both strategies rely on statistical averaging, over a population in the case of epidemiology, and over parameter variability in the case of molecular biology. This use of averages is necessary, yet it is at odds with the idea of personalization. The transition from averaged to personalized predictions requires additional scientific tools, and at least some of these are provided by computational systems biology. The strategy is the following (Fig. 1). Presently, epidemiology yields hypotheses regarding potential risk factors for disease. Molecular biology identifies mechanisms associated with disease risk, and biochemical and physiological techniques describe the mechanisms quantitatively. The findings from these activities lead to conceptual models that are tested in clinical trials. Experimental systems biology greatly enhances this approach with much more comprehensive data than has been possible heretofore. Com- 


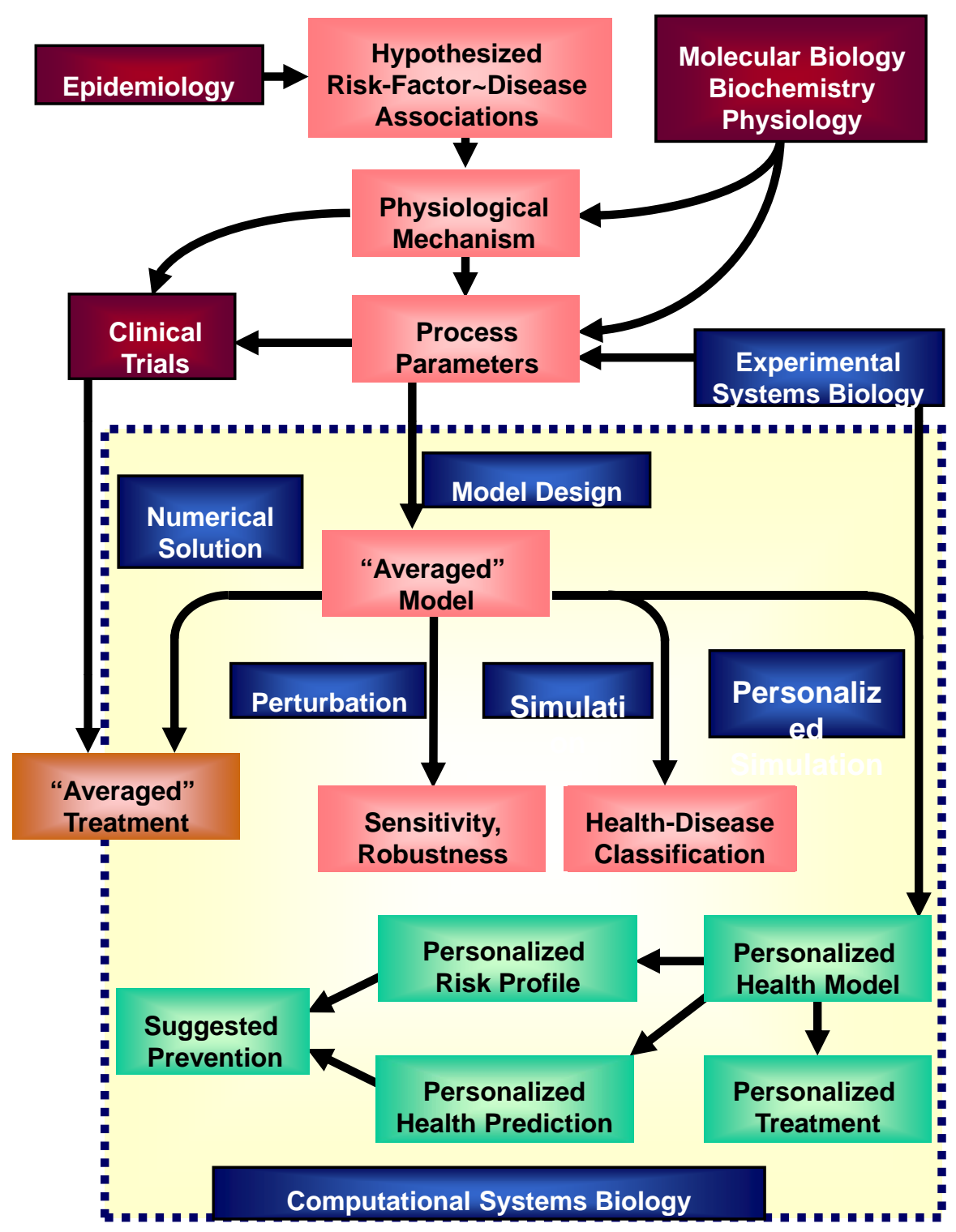

Fig. (1). Traditional and systems biology based paths of medical discovery. Blocks in burgundy color indicate traditional fields contributing to our understanding of disease. The direct results of these contributions are presented in rose, and the ultimate outcome of an averaged treatment strategy is shown in dark orange. Blue blocks show the contributions of experimental and computational systems biology. Computational techniques and results are shown inside the pale yellow box. The results of combining systems biology with traditional approaches are given in green: In addition to the presently used "averaged" approaches, computational systems biology has the potential of "personalizing" predictions of health and treatment of disease.

putational systems biology integrates all this information into mathematical models that describe the average progression toward disease with approximate accuracy [5]. If constructed and implemented correctly, these models are dynamic descriptions of an "averaged" biological system, which may be prone to disease. The value for predictive and personalized health emerges from the fact that these models extend beyond averages. The models allow simulations of the role of every parameter or process, and of synergisms among parameters. Each simulated parameter alteration can be thought of as "inter-personal variation" in gene expression, enzyme activity or signal amplification, lifestyle, or environment that affects one or more components of the system: The computational model can be personalized.
As a first analysis, the model quantifies health, i.e., the robustness of the organism against disease. Simulations where all parameter values are systematically or randomly varied within their typical ranges characterize outputs corresponding either to health or disease. If the model is a valid reflection of the natural system, then a comprehensive simulation analysis can theoretically elucidate every possible health or disease state. Only rarely will varying a single parameter result in disease, and health will be the output of the vast majority of simulations. However, certain specific combinations of changes, even though each one is within the normal range, will push the system out of the acceptable health range and into disease. These generic perturbation studies measure the distance between health and disease, 
provide objective quantification for health disparities, and define the possible trajectories that render a formerly healthy individual ill. A variation on these simulations allows the exploration of personalized risk predictions as well. Suppose that several biomarkers are measured in an individual. Those data are entered into the model for the individual under investigation, and parameters not known for this individual are set as population averages by default. The average default values are now subjected to simulation studies defining the best-case, worst-case, and most likely health risks for this particular individual. The results might, for instance, caution the person to avoid exposures to specific chemicals, based on her or his cytochrome P450 enzyme profile or other personal characteristics the predictive importance of which would not be evident from individual measurements apart from the context of the system. Such models could ultimately determine personalized treatment strategies and dosage regimens.

At this point, most of our models are not at a level of detail and completeness where predictions are always reliable. Nevertheless, extrapolating recent trends in modeling, system identification, and computational analysis, it seems reasonable to expect that models in the foreseeable future will be capable of representing disease phenomena with sufficient reliability. For instance, computational models of the heart [6] and its diseases have reached a level of sophistication that already allows accurate descriptions of infarctions and other disease processes. Similarly, clearly defined molecular and cellular diseases will be approachable with mathematical methods in the not too distant future. The models will integrate all personal health information, compare it with general information and against a growing disease database, and suggest treatment options, which are accompanied by probabilities of success. Obviously, even the prediction of highly probable success may not always come true, but this "surprising" failure is generically no different from today's prognoses by physicians. Moreover, the computational analysis and proposed action will merely be suggestions that will aid the physician in his or her decisions.

\section{CONCLUSIONS}

The shift from treating disease to maintaining health will occur gradually and over a long period of time. Arguably a greater challenge than creating mathematical models of complex diseases is the required shift in thinking about potential future disease among the general population, health care providers and politicians. However, faced with increasing costs and few other alternatives, the prospect of cheap computer-aided personalized diagnoses, prognoses, and treatments will become more and more appealing, especially once the models prove themselves in the real world. While model-based medicine may sound futuristic, the path toward applications of computational systems biology to improvements in personalized and predictive medicine is rapidly emerging. Realizing the potential will require patience and significant investment of human, computational, and material resources, but the rewards will be great.

\section{REFERENCES}

[1] Kitano H. Computational systems biology. Nature 2002; 420(6912): 206-10.

[2] Hood L. Systems biology: integrating technology, biology, and computation. Mech Ageing. Dev 2003; 124(1): 9-16.

[3] Weston AD, Hood L. Systems biology, proteomics, and the future of health care: toward predictive, preventative, and personalized medicine. J Proteome Res 2004; 3(2): 179-96.

[4] Collins CD, Purohit S, Podolsky RH, et al. The application of genomic and proteomic technologies in predictive, preventive and personalized medicine. Vas Pharmacol 2006; 45(5): 258-67.

[5] Voit EO. Computational analysis of biochemical systems: a practical guide for biochemists and molecular biologists. Cambridge, UK, Cambridge University Press; 2000.

[6] Crampin EJ, Halstead M, Hunter P, et al. Computational physiology and the physiome project. Exp Physiol 2004; 89(1): 1-26. 